\title{
La reprise et le défigement des aphorisations dans la presse : mise en évidence de transferts citationnels à travers le temps et l'espace
}

\author{
Grégoire Lacaze \\ Aix-Marseille Université, LERMA EA 853 \\ gregoire.lacaze@univ-amu.fr
}

\begin{abstract}
Résumé. Cette étude se propose de mettre en perspective le défigement de certaines aphorisations à partir d'un corpus constitué d'articles de presse parus dans le quotidien français Le Monde. Cette recherche interroge le travail du journaliste dans la reprise et le défigement de la citation originelle sous l'effet de sa circulation et de son inscription dans le temps historique. L'approche théorique adoptée ici s'inscrit dans la lignée de travaux récents menés en analyse du discours par D. Maingueneau (2012) et A. Krieg-Planque $(2011,2015)$ notamment. Dans ce corpus comportant plus de cinq cents articles, les aphorisations qui font l'objet d'une étude détaillée montrent différentes formes de reprise et de défigement à partir d'une production verbale authentique ancrée dans l'Histoire ou dont l'attribution à une source énonciative connue est hautement probable. Cette étude décrit d'abord le statut de la citation dans la presse et présente les différentes petites phrases et aphorisations que l'on y rencontre. Elle montre ensuite comment la circulation d'une aphorisation au cours de ses réénonciations successives peut conduire à son défigement. L'analyse détaillée de ces phénomènes communicationnels à l'œuvre dans la presse française à partir des observables du corpus de recherche met au jour comment certains types de défigement impliquent des transferts citationnels d'un genre à l'autre et/ou d'une langue à l'autre sous l'effet du temps historique.
\end{abstract}

\begin{abstract}
This research questions the journalist's strategy in the process of rephrasing and altering an original citation under the influence of its circulation and through the passing of time. The theoretical approach adopted here is inspired by some recent research in discourse analysis by D. Maingueneau (2012) and A. Krieg-Planque $(2011,2015)$. Within the corpus gathering more than five hundred articles, the aphorisms under analysis illustrate various types of reduplication and alteration with the assumption that genuine verbalization has occurred at some moment in History or that verbalization is very likely to have happened. This study first describes the status of quotation in the press and it lists the various types of "sound bites" and aphorisms that can be read in newspapers. It then focuses on how the circulation of an aphorism contributes its alteration under the effect of its various reduplications. The detailed analysis of these communicational phenomena encountered in the French press is based on the study of some aphorisms included in the corpus and it aims at identifying certain types of alteration that imply quotational transfers from one genre to another and/or from one language to another under the influence of historical time.
\end{abstract}




\section{Introduction}

La presse, lieu de circulation des discours par excellence, met en dialogue de manière privilégiée les opinions de divers locuteurs rapportés en convoquant leurs propos au sein des articles. Ce faisant, l'activité de citation et la reformulation des paroles d'autrui avant l'insertion dans un article de presse sont au centre de la production journalistique.

Les citations rapportées dans la presse sont de natures très diverses : des citations authentiques, des paroles rapportées à l'origine incertaine, en passant par des discours convoqués mais non authentifiés, jusqu'à des occurrences de discours rapporté témoignant de la non-réalisation événementielle d’un acte énonciatif dans le monde extralinguistique. Cette pluralité de citations dont les statuts énonciatif et pragmatique varient au gré des visées communicationnelles poursuivies par les journalistes témoigne du foisonnement et de la plasticité des pratiques citationnelles.

L'analyse de l'emploi d' "aphorisations » (Maingueneau, 2012) dans la presse illustre la circulation vivante et dynamique de ces fragments citationnels qui peuvent se modifier au cours de leurs réénonciations successives. Cette étude se propose de mettre en perspective le défigement de certaines aphorisations à partir d'un corpus constitué d'articles de presse parus dans le quotidien français Le Monde.

Cette recherche interroge le travail du journaliste dans la reprise et le défigement de la citation originelle sous l'effet de sa circulation et de son inscription dans le temps historique. L'approche théorique adoptée ici s’inscrit dans la lignée de travaux récents menés en analyse du discours par D. Maingueneau (2012) et A. Krieg-Planque $(2011,2015)$ notamment.

Dans ce corpus comportant plus de cinq cents articles, les aphorisations qui font l'objet d'une étude détaillée montrent différentes formes de reprise et de défigement à partir d'une production verbale authentique ancrée dans l'Histoire ou dont l'attribution à une source énonciative connue est hautement probable.

Cette étude décrit d'abord le statut de la citation dans la presse et présente les différentes petites phrases et aphorisations que l'on y rencontre. Elle montre ensuite comment la circulation d'une aphorisation au cours de ses réénonciations successives peut conduire à son défigement. L’analyse détaillée de ces phénomènes communicationnels à l'œuvre dans la presse française à partir des observables du corpus de recherche met au jour comment certains types de défigement impliquent des transferts citationnels d'un genre à l'autre et/ou d'une langue à l'autre sous l'effet du temps historique.

\section{Les citations dans la presse, leur circulation et leur défigement}

Les citations que les journalistes insèrent dans leurs articles sont de natures et d'extensions diverses. Elles peuvent évoquer des actes énonciatifs authentiques ou à l'origine énonciative incertaine mais elles peuvent également représenter un discours qui n’a pas été tenu et qui est simplement envisagé comme une alternative au discours attesté. 
La citation authentique est, par nature, localisable dans un environnement spatio-temporel. Ainsi, l'acte d'énonciation d'origine d'une citation donnée est généralement traçable dans le temps et dans l'espace. Il est alors possible de dater cette prise de parole en l'inscrivant dans le temps historique et en la localisant dans un espace géographique donné. Par ailleurs, de nombreux chercheurs ont relevé qu'une citation renvoie souvent à un «événement historique » (Neveu et Quéré, 1996, Quéré, 2013, Londei et al., 2013) du monde extralinguistique. Elle peut alors être rattachée à un «événement discursif » inscrit dans un « instant discursif » ou un « moment discursif » (Moirand, 2007).

\subsection{Les « petites phrases » et les différents types d'aphorisations}

Plusieurs définitions cohabitent pour désigner ce que sont les «petites phrases » (voir Brasart, 1994, Krieg-Planque et Ollivier-Yaniv, 2011 notamment). Dans la définition qu'en donne Le Trésor de la Langue Française Informatisé, la « petite phrase » est un " propos bref d'un homme politique, qui sert à frapper l'opinion ».

A. Krieg-Planque (2011: 26) la définit ainsi : une «"petite phrase" est un syntagme dénominatif métalinguistique non-savant (et plus précisément : relevant du discours autre approprié), qui désigne un énoncé que certains acteurs sociaux rendent remarquable et qui est présenté comme destiné à la reprise et à la circulation ».

Dans cette étude, la petite phrase peut être émise par un acteur de la scène politico-médiatique ou par un locuteur dont les propos tenus sont jugés utiles d'être repris par les médias. La petite phrase est donc généralement une citation authentique associée à un acte énonciatif unique et datable.

À côté des petites phrases qui reproduisent les dires de locuteurs cités, distingués par les acteurs médiatiques et dont les discours entrent en circulation, les aphorisations obéissent, à un régime énonciatif spécifique, comme l'a montré D. Maingueneau (2012). Plusieurs aphorisations sont identifiables et la taxinomie adoptée par le linguiste est la suivante: "aphorisations primaires », "aphorisations secondaires » et «particitations » ${ }^{1}$ (Maingueneau, 2012). Les «aphorisations "primaires" » incluent " proverbes, adages, devises, slogans... " (Maingueneau, 2012 : 23). Les « aphorisations secondaires » impliquent, par essence, la cohabitation de « deux contextes effectifs : un contexte source, un contexte d'accueil » (Maingueneau, 2012: 25). Les particitations ${ }^{2}$, qui sont, à l'origine, des aphorisations secondaires, ont acquis au cours du temps une grande notoriété et, par leur portée historique, sont entrées dans un Thesaurus. Elles ne constituent pas un ensemble homogène et des sous-catégories peuvent être mises au jour : les «aphorisations sentencieuses ${ }^{3}$ (Maingueneau, $2012: 61$ ) comme les proverbes et adages, les « particitations de groupe » (2012: 63) comme les slogans de groupes constitués et les slogans commerciaux, les «particitations scripturaires » (2012: 67) qui correspondent aux citations d'hommes célèbres et qui sont entrées dans l’Histoire.

Voici quelques exemples de particitations scripturaires : «Je vous ai compris », particitation prononcée par le Général de Gaulle en 1958, «I have a dream », particitation prononcée par Martin Luther King en 1963, " I want my money back », particitation attribuée à Margaret Thatcher dans un discours prononcé en 1979. D’autres aphorisations sont également entrées dans l'Histoire comme « Yes, we can », slogan de la campagne de Barack Obama en 2008 et qui pourrait être envisagé comme une particitation de groupe : le slogan du candidat Barack Obama et, par extension, le slogan du parti démocrate soutenant son candidat à la présidentielle américaine. À propos de telles aphorisations, A. Krieg-Planque (2011: 36) souligne la « concision » des "phrases historiques telles que I have a dream » qui sont immédiatement identifiables et rattachables à un événement historique majeur dans l’histoire de l’humanité.

\subsection{La citation inscrite dans la mémoire collective et historique et l'exploitation d'un « Thesaurus cognitif »}

Envisager l'inscription d'une citation dans la «mémoire discursive " ${ }^{4}$ et patrimoniale, c'est analyser avant tout le statut énonciatif de paroles attribuées à une source énonciative. Il s’agit alors d'évaluer les 
conditions effectives de production verbale de l'acte de parole rapporté. Ces considérations amènent à interroger la localisation de l’événement discursif, associé à cette citation, qui doit pouvoir être repéré par ses coordonnées spatio-temporelles.

À partir des traces citationnelles qu'il peut identifier dans un article de presse, l'analyste de discours cherche à retracer la genèse de la prise de parole du locuteur cité à partir des données objectives fournies dans l'article mais aussi à partir de son expérience personnelle de citoyen, notamment grâce à la mémoire patrimoniale et culturelle de la société dans laquelle il vit.

A. Krieg-Planque (2011 : 35) rappelle que « la question des énoncés faits pour être retenus est ancienne : dès la rhétorique antique, est mise en place la notion de memoria, qui met l'accent sur les figures et formes favorables à la mémorisation et à la reprise ». Elle précise que «la memoria correspond à cette partie de la rhétorique qui s’intéresse aux procédés permettant de mémoriser un discours » (Krieg-Planque, 2011 : 35).

L'analyste de discours, qui recherche à partir d'une aphorisation les traces de l'inscription d'une citation historique dans la mémoire patrimoniale, cherche ainsi à évaluer le degré de «mémorabilité » ${ }^{5}$ de l'énoncé historique. En effet, toute citation est intrinsèquement liée aux conditions de sa production et notamment à la problématique de son inscription dans le temps chronologique.

Les citations authentiques, en fonction de leur portée historique, vont ou non s’inscrire dans la mémoire patrimoniale et pouvoir compléter un stock phraséologique et lexical dans lequel tout journaliste pourra puiser et prolonger ainsi le «cycle de vie » d'une citation plus ou moins enfouie dans la mémoire collective.

Le journaliste de presse et/ou le locuteur rapporté, qui choisissent de convoquer des propos attribués à une source énonciative tierce, participent à la réactivation et/ou à la perpétuation de la notoriété d'une citation donnée à travers le temps historique. Pour ce faire, l' «emprunteur » d'une petite phrase ou d'une aphorisation, fait usage d'un « Thesaurus cognitif » qu'il suppose partagé avec son interlocuteur/lecteur. La connaissance par les lecteurs de la presse de ce Thesaurus propre à une société donnée est bien un présupposé sur lequel s’appuie le journaliste emprunteur.

Ce Thesaurus, dont les contours et la composition ne sont pas figés, évolue au fil du temps et s'enrichit sans cesse de nouvelles petites phrases, au fur et à mesure que les acteurs politiques, institutionnels, littéraires, scientifiques, sportifs et sociétaux prennent la parole et que leurs discours circulent à travers la société.

Deux stratégies discursives sont possibles pour celui qui exploite ce Thesaurus : soit le journaliste, par souci de pédagogie, signale la source de la citation originelle (c'est le cas d'une aphorisation secondaire), soit il estime que l'aphorisation est universellement connue (c'est une particitation scripturaire) et l'origine énonciative est masquée. Dans ce second cas, le lecteur peut ne pas reconnaître qu'il s'agit d'une aphorisation exhumée du Thesaurus, s’il n’a pas la connaissance de cette citation.

\subsection{La circulation des aphorisations : de la fidélité au défigement}

La circulation d'une citation va dépendre de son type et de son contexte d'énonciation. Ainsi, lorsque la citation d'un locuteur cité ne présente pas, selon les médias, un intérêt suffisant, elle pourra n'être mentionnée qu'une seule fois dans la presse et ne donnera pas lieu à des reprises ultérieures. Au contraire, une citation assimilée à une petite phrase ${ }^{6}$ connaîtra elle une circulation bien plus importante et pourra faire l’objet de multiples réénonciations avec ou sans déformation.

Par essence, les «petites phrases », envisagées comme un «phénomène de coproduction discursive » (Krieg-Planque et Ollivier-Yaniv, 2011 : 19), impliquent une « circulation médiatique » (2011 : 21). Cette circulation s'inscrit elle aussi dans la "ronde des discours produits et transmis par les médias » (Moirand, 2007 : 4) en raison de leur portée événementielle. Quand une petite phrase (souvent une aphorisation) est reprise abondamment dans les médias, l'on assiste à un «phénomène de 
"panaphorisation" » (Maingueneau, 2012 : 88) défini ainsi : un « terme qui combine "aphorisation" et le pan- de "pandémie" ».

Par leur statut même, les particitations scripturaires, qui désignent des " citations célèbres » (Maingueneau, 2004 : 115), sont fréquemment reprises par les journalistes car elles appartiennent à la mémoire doxique et sociétale. Elles reposent sur une complicité relationnelle entre les journalistes et les lecteurs, ce qui suppose un partage de références historiques et culturelles communes.

Envisager la circulation d'une aphorisation, c'est aussi prendre en compte la reprise et la réénonciation d'autres aphorisations comme les parémies, qui constituent un vaste corpus dans lequel le locuteur emprunteur puise pour reprendre et, le cas échéant, défiger ces énoncés canoniques.

La reprise d'une citation peut conserver à l'identique la séquence citationnelle effectivement verbalisée. Toutefois, la mise en circulation de citations par la réénonciation multiple de paroles authentiques peut donner lieu à une transformation plus ou moins importante de la séquence citationnelle d’origine.

Dans le cas d'une particitation, qui appartient au patrimoine historique et discursif, le journaliste n'a pas besoin de rappeler les conditions de l'acte énonciatif d'origine. Le lecteur mobilise ses connaissances et, comme la particitation est inscrite dans la mémoire doxique, il est le plus souvent en mesure d'associer implicitement cette citation à un événement historique entré dans l'histoire d'une nation ou d'un pays. Ces particitations s'apparentent à une parole sentencieuse et peuvent donner lieu à des déformations, des variantes. Cependant, le lecteur doit pouvoir reconnaître l'écho de la citation originelle, ce qui met en exergue ici la stratégie et la visée énonciatives du locuteur rapporteur exploitant les notions d'intertextualité, de dialogisme et de polyphonie à l’œuvre dans ces paroles circulantes ou « circulèmes ${ }^{7}$.

Par ailleurs, la particitation lorsqu'elle a le statut de « petite phrase » se voit souvent teintée d'une valeur illocutoire car « la "petite phrase" identifiée comme telle est supposée correspondre à un certain acte de langage: promesse, engagement, soutien, exigence, exhortation, menace, condamnation, reniement, offense, demande d'excuse... » (Krieg-Planque, 2011 : 36).

\subsection{Le « défigement » comme concept, ses ressorts et ses conséquences}

Le concept de «défigement » et, par opposition, celui de «figement » mobilisent depuis plusieurs décennies l'intérêt des linguistes. P. Fiala et B. Habert (1989) ont analysé le « défigement » affectant les titres de la presse française. Adoptant le point de vue de la « sémantique interprétative », F. Rastier (1997) a étudié les phénomènes de "figement » et de "défigement » des lexies. Il y souligne le caractère « subversif » du « défigement des locutions ». Le locuteur, en devenant l'auteur d'un défigement, tente de s'affranchir des règles et « normes qui ont présidé au figement » mais il peut également exploiter l' « effet ludique » de ce défigement (Rastier, 1997 : 310). Il semble tout à fait envisageable de transposer cette analyse au défigement des aphorisations qui contiennent des lexies et locutions.

Dans son étude portant sur les discours d'autorité, A. Krieg-Planque (2015) envisage elle aussi le défigement comme une «subversion » du figement. Selon A. Krieg-Planque (2012 : 97), le " figement » est défini comme un "espace de resserrement de la créativité verbale ». Le figement a fait l'objet de nombreuses autres études récentes, comme les ouvrages dirigés par J.-C. Anscombre et S. Mejri (2011) et L. Perrin (2013). Le défigement évoqué dans notre étude est donc avant tout caractérisé par son aspect transgressif et subversif. Il est proche du concept de « détournement » adopté par S. Leroy (2005).

L'altération d'une aphorisation peut intervenir à différents niveaux. F. Rastier (1997) évoque le défigement des «lexies simples » et «complexes » et les «substitutions paradigmatiques » souvent à l’œuvre. Une étude menée par R. Galisson (1993) détaille les diverses transformations affectant ce qu’il appelle des «palimpsestes verbaux ». Il envisage, parmi les divers "modes de délexicalisation », la " délexicalisation avec ou sans filiation phonique » et la "délexicalisation avec ou sans déstructuration syntaxique » (1993: 45-46). Ces modes de délexicalisation sont exploités par les journalistes lors du défigement des aphorisations. 
Le défigement peut opérer à plusieurs niveaux énonciatifs : en effet, il peut être à l'initiative du journaliste ou à l'initiative du locuteur rapporté. Dans le premier cas, il s'agit de la reprise d'une citation issue d'un Thesaurus que le journaliste va déformer avec une visée pragmatique et stylistique. Dans le deuxième cas, un locuteur emprunteur défige une citation et le journaliste se contente de reprendre et rapporter les propos du locuteur «défigeur ». Le défigement de citations authentiques va montrer la circulation de la parole historique sous la forme d’une chaîne récursive avec des "énonciateurs rapportants mis en abîme » (Rosier, 2005 : 107). Ainsi, une particitation historique peut engendrer diverses déformations palimpsestiques qui se démultiplient au gré des reprises successives par une « chaîne d'énonciateurs rapportants » illustrant une « forme de la récursivité » (Rosier, 2005 : 104).

Cette recherche se propose d'interroger les motivations qui conduisent à une opération de défigement. Lorsque le journaliste prend la position d'un locuteur défigeur, il semble que l'effet de sensationnalisme et la "pulsion ludique ${ }^{8}$ avec ou sans valeur parodique constituent les motivations principales d'un défigement.

L’opération de défigement d'une citation présuppose une bonne connaissance historique chez le lecteur afin qu'il puisse reconnaître l'intertextualité à l'œuvre dans l'article contenant une citation plus ou moins retravaillée par le journaliste ou l'équipe de rédaction.

Le détournement d'une citation patrimoniale permet au journaliste de «défiger un énoncé sur le mode plaisant » afin d' « établir la connivence par l'activation d’une mémoire » (Krieg-Planque, 2012 : 193). Ce faisant, le journaliste cherche à « établi[r] avec le lecteur une relation de connivence » mais celle-ci n'est pas garantie si « l'allusion n’a pas été reconnue comme telle » par le lecteur (Krieg-Planque, 2012 : 194).

En tant qu'acteur médiatique et membre du corps social au même titre que ses lecteurs, le journaliste est amené à écrire ses articles en fonction de contraintes temporelles imposées par les médias ; il vit donc lui aussi dans « la tyrannie de l'instant ${ }^{9}$. En conséquence, le journaliste peut « oublier » de recontextualiser l'aphorisation qu'il défige et ne pas fournir au lecteur des informations essentielles à sa compréhension.

Si le lecteur n’a pas la connaissance de l'événement à la grande portée historique auquel fait référence la citation, il y a un échec partiel de la réception de l'aphorisation. En effet, comme l'évoque A. Krieg-Planque (2012 : 191) : «Certains énoncés ne fonctionnent qu'en tant qu’ils sont rattachés à d'autres énoncés par rapport auxquels ils constituent des allusions. Le discours en train de se faire ne déploie alors ses effets de sens que si est activé, en mémoire, un lien avec une autre énonciation ». Dans ce cas, le lecteur ne parvient pas à lire en filigrane la citation originelle, le processus de défigement devenant alors producteur d’opacité énonciative.

Le défigement d'une citation joue pleinement sur la complicité relationnelle entre le journaliste et son lectorat, c'est-à-dire sur l'existence d'une connivence dialogique entre l' « énonciateur-rapporteur » et l' «énonciataire-lecteur » dans l'approche praxématique du dialogisme introduite par J. Bres et B. Verine (2002) et reprise par S. Leroy (2005 : 203) :

[...] l'énoncé dialogique présente un dédoublement énonciatif en deux systèmes d'énonciation distincts et hiérarchisés. Ainsi, l’énoncé actualisé (É) constitue l'énonciation enchâssante, l'énonciation enchâssée faisant entendre la parole représentée (é) ; à ces deux énonciations se rattachent deux énonciateurs et deux énonciataires, $E_{1}$ et $E_{2}$ pour l'énonciation enchâssante, $e_{1}$ et $e_{2}$ pour l'énonciation enchâssée.

Des « faits de parole » (Perrin, 2001 : 148, cité par Leroy, 2005 : 209) attestés qui « sont ensuite repris et inscrits dans une chaîne de reprises citatives " (Leroy, 2005 : 209) conduisent à des phénomènes de « sédimentation discursive » (2005 : 210), comme en témoignent les déformations, les défigements et les détournements observés dans les titres de presse. 


\section{Le défigement des aphorisations comme catalyseur de transferts citationnels à travers le temps historique, le genre et l'espace géographique}

Le locuteur emprunteur (qu'il soit journaliste ou non) qui choisit à dessein de reprendre une aphorisation peut reproduire à l'identique l' «énoncé aphorisé " ${ }^{10}$, mais il peut aussi, apporter sa «touche personnelle » à l'aphorisation originelle, participant ainsi au processus de défigement. Son investissement personnel dans le défigement repose sur sa propre subjectivité et sur la visée perlocutoire qu'il poursuit, ce défigement pouvant entraîner des transferts citationnels qui s’affranchissent des frontières génériques et/ou géographiques avec une prise en compte du temps historique, comme le révèlent les observables du corpus.

\subsection{De la réénonciation à l'identique d'une citation historique à son détournement}

Le journaliste peut « reprendre un énoncé à l'identique » (Krieg-Planque, 2012 : 192). Il se contente alors de reproduire une citation connue :

«I want my money back » (Le Monde 07/11/2013)

La séquence historique «I want my money back » attribuée à Margaret Thatcher correspond en fait à une version reformulée ${ }^{11}$ des propos effectivement prononcés le 30 novembre 1979 à Dublin par le premier ministre britannique, lors d'une conférence de presse d'un sommet européen, comme en atteste la transcription de la conférence de presse disponible sur le site Internet de la Margaret Thatcher Foundation. Cette "séquence figée ", comme pourrait la qualifier A. Krieg-Planque (2009 : 73), existe bien en tant qu'aphorisation. Cette citation attribuée à Margaret Thatcher est inscrite dans la mémoire patrimoniale. Elle a fait l'objet d'une circulation relativement importante dans la presse avec de multiples réénonciations depuis 1979.

Cette petite phrase entre dans la catégorie que les analystes du discours anglo-saxons qualifient de "sound bite " ${ }^{12}$ et qui, selon D. McCallam (2000 : 52-53) se diffuse dans «la conscience politique américaine [...] au cours de l'élection présidentielle de 1988 ».

Dans l'énoncé étudié, il y a une double « opération de décontextualisation et de recontextualisation » de la citation historique (Krieg-Planque, 2012 : 192). L'attitude des Français qui rechignent à payer des impôts est comparée au point de vue énoncé en son temps par Margaret Thatcher, comme cela est mentionné dans le corps de l'article :

À les entendre protester contre leur avis d'imposition, on les prendrait pour Thatcher.

«I want my money back! », serinait la « Dame de fer » contre l’Europe. (Le Monde

07/11/2013)

Une citation historique peut être relativement productive en donnant lieu à des réénonciations multiples et tout locuteur rapporteur peut détourner de manière parodique la citation originelle. Alors qu’A. Krieg-Planque (2009 : 73) a montré la possibilité du « défigement » d'une formule sous l'effet de sa circulation, l'on peut assister de manière analogue au défigement d'une citation mais celui-ci doit rester limité afin que le destinataire de la citation, en l'occurrence le lecteur, puisse reconnaître dans un processus de lecture palimpsestique la citation originelle qui est gravée dans le patrimoine doxique. En effet, le titre d'article de presse semble être le lieu privilégié du « détournement » qui est « un phénomène purement dialogique », comme l’envisage S. Leroy (2005 : 202).

Le journaliste effectue ici un transfert citationnel d'un pays à l'autre (de la Grande-Bretagne à la France) en utilisant une langue étrangère (l'anglais) dans un quotidien de langue française.

La citation historique précédente a subi un défigement parodique dans un autre article du Monde :

I want my UK back ! (Le Monde 23/05/2014) 
L'aphorisation est accompagnée du nom de l'aphoriseur, Nigel Farage, leader du parti UKIP au Royaume-Uni et d'une photo de cet homme politique prononçant un discours à la tribune lors d'une réunion politique à Londres en mai 2014 pendant la campagne des élections européennes. L'analyse du contenu de l'aphorisation révèle la nature du défigement parodique opéré par le journaliste et non pas par l'homme politique.

Dans le titre de l'article du Monde, il y a une substitution paradigmatique du trochée historique money par le trochée UK mais le cadre prosodique est conservé. En convoquant une citation historique qu'il détourne, le journaliste participe au processus de défigement. Il fait entendre une énonciation à plusieurs niveaux. Il est à noter qu'il est assez remarquable que ces aphorisations en anglais apparaissent dans un quotidien français. Par ailleurs, il est intéressant de constater que le journaliste prête à Nigel Farage des propos qu'il n'a pas tenus. Il dresse une analogie entre les ambitions de l'homme politique britannique qui souhaite que son pays retrouve une plus grande souveraineté vis-à-vis de l'Union Européenne et l'attitude passée de Margaret Thatcher qui exprimait ses réticences à l'idée que le Royaume-Uni verse à l'Union Européenne une contribution financière qu'elle jugeait trop importante.

\title{
3.2 Transferts linguistiques d'une langue à l'autre, cumul de défigements et hétéroglossie
}

Le défigement peut parfaitement s’affranchir des frontières géographiques et langagières. Le locuteur défigeur peut ainsi emprunter une aphorisation dans une autre langue que la sienne et procéder ensuite à une opération de détournement.

Il est parfois possible d'assister à une mise en abyme de plusieurs phénomènes de défigement, comme dans l'énoncé suivant :

\begin{abstract}
Au fond, cet article rappelle l'importance de l'hommage rendu aux pionniers et visionnaires de génie. "Si j'ai vu plus loin, c'est en montant sur les épaules de géants », avouait Isaac Newton en 1676. Ce géant reprenait lui-même une métaphore du XII ${ }^{\mathrm{e}}$ siècle, imaginée par Bernard de Chartres : «Des nains sur des épaules de géants. » (Le Monde 03/06/2015)
\end{abstract}

Le journaliste reprend une citation d'Isaac Newton (« If I have seen further, it is by standing on the shoulders of giants ») mentionnée dans une lettre adressée à Robert Hooke en février 1676. Toutefois, la citation originelle a été traduite en français par le journaliste. Il s'agit en quelque sorte d'une première opération de défigement. Le journaliste du Monde a jugé utile de rappeler aux lecteurs la genèse de cette citation historique ; ce qui montre que Newton a été lui aussi un locuteur défigeur. En effet, Newton aurait emprunté cette aphorisation au philosophe français Bernard de Chartres, qui est censé avoir inventé la métaphore latine « Nani gigantum humeris insidentes » ${ }^{13}$.

Le journaliste a choisi délibérément de masquer l’hétéroglossie inhérente à ces aphorisations historiques (du latin au français en passant par l'anglais). Cette «transparence langagière » qui s'affranchit des frontières géographiques et linguistiques est relativement courante dans la presse. L'énoncé suivant illustre ce point :

Mais arrivera bien un moment où David Cameron devra présenter à ses électeurs ce qu'il a obtenu des Européens. Comme disait également Lénine : les faits sont têtus. (Le Monde 31/05-01/06/2015)

Le journaliste rapporte une citation attribuée à Lénine, qui l'a prononcée en russe en octobre $1917^{14}$ : « Факты - упрямая вещь » ${ }^{15}$.

Parfois, le journaliste tient à «faire entendre » la voix du locuteur cité en reproduisant l'aphorisation allogène et en l'accompagnant d'une traduction en français :

Avec succès, puisque sur les conseils de Charles Lindbergh la compagnie aérienne américaine Pan Am décidait d'acheter 200 Mystère 20, le premier jet de ce qui deviendra la famille Falcon. «i’ve found our bird» («j'ai trouvé notre oiseau »), 
aurait télégraphié le pionnier américain de l'aviation aux dirigeants de la compagnie américaine. (Le Monde 03/06/2015)

Le message censé avoir été télégraphié par l'aviateur américain Charles Lindbergh est retranscrit pour produire un effet d'authenticité de la parole rapportée. Le conditionnel du verbe introducteur télégraphier ${ }^{16}$ employé ici porte la " valeur médiative de ouï-dire » (Bres, 2014 : 23).

Dans les deux énoncés ci-dessus, il y a bien reprise d'une aphorisation allogène et adaptation de celle-ci à la langue cible (le français). Il s'agit d'une certaine forme de défigement : l'opération de traduction qui assure le transfert citationnel d'une langue à l'autre est bien la trace d'une subjectivité assumée par le journaliste emprunteur. Un contenu propositionnel initialement verbalisé dans une langue donnée peut ainsi circuler dans le monde physique et être repris, voire défigé dans une langue autre.

La circulation d'une aphorisation au-delà de sa zone géographique originelle et sa diffusion, sa dispersion, à travers l'espace national d'abord et l'espace mondial ensuite, soulignent alors la portée universalisante d'une telle citation.

Lorsque le défigement est opéré par le journaliste «emprunteur ", l'acte de parole attribué à un énonciateur physique ne correspond généralement pas à un acte authentique :

«Qui d'autre? » pourrait demander George Clooney... (Le Monde 07/10/2013)

Il y a reprise par le journaliste du slogan publicitaire de Nespresso (What else ?), qui est une particitation de groupe et dont l'acteur américain est l'aphoriseur emblématique de la marque de machines à café. Le journaliste traduit dans un premier temps cette aphorisation (Quoi d'autre ?) et effectue ensuite la substitution paradigmatique d'un pronom interrogatif par un autre pour aboutir à l'énoncé défigé.

L'aphorisation défigée se présente au niveau syntaxique comme une occurrence de discours direct mais il s'agit en fait d'un " pseudo-discours rapporté », comme l'appelle L. Rosier (2008: 19), qui appartient à la famille des énoncés de «discours représenté » (2008: 4). L'emploi du verbe pouvoir dans le «segment contextualisant ${ }^{17}$ devant le verbe demander désactualise la question retranscrite par le journaliste.

\subsection{La reprise et/ou le défigement d'une parémie d'une langue à une autre}

Les proverbes et adages, en tant qu'aphorisations sentencieuses, font partie de la culture populaire et constituent des repères communs à une société donnée. Il est donc naturel que les journalistes choisissent souvent de citer un proverbe, se laissant la possibilité éventuellement de le défiger. Comme pour les autres aphorisations, la reprise et/ou le défigement d'une parémie peuvent être à l'initiative du locuteur rapporté ou du journaliste-locuteur rapporteur. Ces opérations peuvent intervenir à l'intérieur d'une langue donnée ou bien impliquer le passage d'une langue à l'autre.

Dans l'énoncé ci-dessous, le journaliste emprunteur se contente de reprendre une certaine forme de parémie employée pour parler du cours d'une action en bourse :

Les arbres ne montent pas jusqu'au ciel, dit un adage autrefois bien connu des boursiers. (Le Monde 08-09/03/2015)

Autrement dit, cet adage signifie que le cours d'une action donnée ne peut connaître une hausse continue sans connaître parfois des chutes. L'aphorisation est reproduite ici sans faire l'objet d'un défigement.

Le défigement peut affecter aussi le contenu propositionnel d'une maxime avec la substitution paradigmatique d'un nom présent dans le proverbe par un autre :

«Toute connexion mérite salaire », voudrait la nouvelle maxime du travail numérique avancée par le site Lettreducadre.fr, qui préconise l'instauration d' «une trêve des mails ». (Le Monde 02/10/2014)

À partir du proverbe " Tout travail mérite salaire », le journaliste emprunteur défige le proverbe à des fins argumentatives en choisissant le terme connexion qui appartient au champ sémantique du numérique, dont il est question dans l'article. 
Les proverbes en langue latine en tant qu'aphorisations sentencieuses font partie du Thesaurus collectif qu'un locuteur emprunteur peut exploiter :
«De minimis non curat praetor» («Le juge ne s'occupe pas des choses insignifiantes $»$ ), a répondu, dimanche 29 mars, le cabinet du premier ministre à M. Van Rompuy. Le clin d'œil était appuyé - M. De Wever raffole des citations latines - mais n’a pas calmé la polémique. (Le Monde 01/04/2015)

Le locuteur emprunteur qui se contente de réénoncer une aphorisation latine est un membre du cabinet du premier ministre belge. La traduction proposée par le journaliste ou l'équipe de rédaction fait généralement l'objet d'un consensus et connaît peu de variations.

Le défigement d'une parémie en langue latine peut être également à l’initiative du journaliste. Celui-ci participe à la circulation d'une aphorisation sentencieuse :

« Dura lex, sed lex », pourrait dire le latex, déprimé, évoquant la dure loi des marchés financiers. (Le Monde 08-09/03/2015)

Le journaliste ne se contente pas de reprendre un proverbe latin bien connu, il opère un défigement de celui-ci non pas au niveau du contenu propositionnel rapporté mais au niveau du type de la source énonciative prenant en charge ce proverbe. Le détournement opéré par le journaliste repose sur le réemploi d'un proverbe latin en l'attribuant cette fois à une source non humaine. Par ailleurs, au niveau stylistique, le proverbe réalisé sous la forme d'un distique (à l'instar du proverbe français Tel père, tel fils analysé par J.-C. Anscombre (2000) dans son étude des structures métriques de la parole proverbiale) prolonge l'effet produit par l'homéotéleute du proverbe en ajoutant un troisième phonème / $\varepsilon \mathrm{ks} /$ dans l'énoncé en jouant sur l'emploi du nom latex en position de « locuteur métonymique ».

L'emploi d'une structure mimétique de celle d'un discours direct authentique produit un effet de sens remarquable, comme si le latex pouvait avoir voix au chapitre. L’analyse de cet énoncé démontre le haut degré d'investissement du journaliste dans l'opération de défigement grâce à l'exploitation des effets phonologiques de l’homéotéleute.

\subsection{La circulation d'une aphorisation secondaire à l'épreuve du temps historique}

La prise en compte de la réalité du monde extralinguistique peut conduire à une réévaluation de la reprise d'une aphorisation secondaire au niveau du temps grammatical affectant le verbe introducteur de la citation (lorsqu'un verbe est présent dans le segment contextualisant).

Comparons à cet effet deux citations similaires convoquées par le même journaliste du Monde dans deux articles sportifs à cinq ans d'intervalle :

Comme dirait Thierry Roland, poète du commentaire sportif, «pas un temps à mettre un footballeur dehors ». (Le Monde 17/09/2009)

"Pas un temps à mettre un footballeur dehors », aurait dit feu Thierry Roland. (Le Monde 01/07/2014)

Célébrant la faconde du journaliste sportif français Thierry Roland décédé en juin 2012, le journaliste Bruno Lesprit reproduit une citation explicitement attribuée à cette figure emblématique du journalisme sportif à la télévision. Il ne nous a pas été possible de déterminer si cette aphorisation a véritablement été énoncée à la télévision par celui censé l'avoir prononcée. Le journaliste du Monde a souhaité convoquer à nouveau cette citation en 2014, cinq ans après l'article de 2009, mais il a changé le conditionnel présent en conditionnel passé car le journaliste sportif est décédé entre temps. "S'exprimer à la manière de » est un artifice auquel les journalistes ont souvent recours. Ce faisant, ils convoquent des discours nécessairement recontextualisés car leur auteur présumé n’a pas tenu les propos en lien avec l'actualité traitée dans l'article. 
Le lien entre temps historique et inscription de la parole dans la mémoire patrimoniale et sociale est mis en évidence dans les deux citations ci-dessus. L'alternance entre dirait ou aurait dit fait intervenir des éléments factuels incluant le degré de plausibilité de la citation. Le plan «toncal », introduit par J. Damourette et É. Pichon (1936) ${ }^{18}$, est bien le plan de la désactualisation énonciative dont le morphème -ait est la trace mais, en plus, le contexte historique vient se greffer aux propos du journaliste en conditionnant le temps grammatical utilisé.

Ces deux énoncés illustrent également la mise en abyme de plusieurs processus de reprise et de défigement. À partir du proverbe "Pas un temps à mettre un chien dehors », Thierry Roland a opéré un défigement pour appliquer ce proverbe au domaine sportif par substitution d'un élément paradigmatique par un autre. Le journaliste emprunteur a, quant à lui, fait circuler cette aphorisation défigée au cours des années.

\subsection{L'intersémioticité possible pour un défigement}

Le défigement n’opère pas nécessairement à l'intérieur d’un genre discursif donné. L'appartenance de l'aphorisation à un Thesaurus favorise le passage et le transfert citationnel d'un genre discursif à un autre. Ainsi, les paroles d'une chanson (comme le refrain, par exemple) peuvent être reprises et détournées dans la presse. Il en va de même pour la citation d'une œuvre littéraire, extraite du corpus textuel d'origine et pouvant donner lieu à une reprise parodique.

Lorsque le journaliste est à l'initiative du défigement, il peut modifier une réplique célèbre d'une pièce du théâtre classique pour apporter une touche d'humour à son article :

\footnotetext{
L'argent «propre » des sponsors va-t-il chasser l'argent «sale » de la corruption ?

Restons prudent. «Couvrez cette corruption que je ne saurais voir », aurait pu écrire

Molière s’il était entré dans un stade de football. (Le Monde 04/06/2015)
}

Ici, le journaliste défige la réplique de Tartuffe s'adressant à Dorine et lui confiant son mouchoir : « Couvrez ce sein que je ne saurais voir » (Molière, Tartuffe III,ii). La présence du verbe pouvoir dans le segment introducteur présuppose la non-réalisation événementielle de l'acte de parole rapporté en association avec le conditionnel passé, qui est un «conditionnel d'éventualité » (Dendale et Kreutz, 2014 : 134).

De manière analogue, le journaliste déforme la réplique attribuée à Rabelais dans « Aux lecteurs » débutant Gargantua :

\section{"Rire est le propre de l'homme connecté », aurait pu écrire François Rabelais si Gargantua avait été abonné à Internet ou avait disposé d’un abonnement de téléphonie mobile. (Le Monde cahier éco\&entreprise 31/01/2014)}

Rabelais écrit :

« Mieulx est de ris que de larmes escripre,

Pour ce que rire est le propre de l'homme ». (Gargantua 1v)

Le journaliste a isolé la séquence devenue proverbe « Rire est le propre de l’homme » et lui a adjoint l'adjectif qualificatif connecté pour rendre cette aphorisation plus actuelle pour un lecteur du XXI ${ }^{\mathrm{e}}$ siècle. L’opération de défigement adoptée ici permet de prolonger l'inscription de l'aphorisation originelle dans l'Histoire et de réactiver son énonciation en la détournant de manière mineure sur un mode parodique et humoristique. C’est aussi une occurrence de pseudo-discours rapporté.

Une citation à l'origine historique douteuse peut néanmoins circuler à travers les siècles et à travers le monde avec comme effet potentiel des altérations successives. Sa reprise dans un journal peut laisser supposer une opération de traduction. L’énoncé suivant illustre cette circulation :

«Il y a trois sortes de mensonges: les mensonges, les gros mensonges et les statistiques », aurait un jour déclaré l’homme d’État britannique Benjamin Disraeli (1804-1881). (Le Monde 18/04/2014) 
L’origine historique de cette citation fait débat. L'auteur américain Mark Twain attribue ces propos au premier ministre britannique Benjamin Disraeli : «There are three kinds of lies: lies, damned lies, and statistics. ${ }^{19}$. L’énoncé du Monde est sous l'influence de l'intertextualité ainsi que de l'effet dialogique et polyphonique.

L'attribution de l'aphorisation est incertaine, comme l'indique l'emploi du conditionnel passé à valeur médiative de ouï-dire. Pourtant, elle est généralement inscrite dans le Thesaurus des citations d'hommes politiques. Le lecteur est le témoin d'une chaîne circulante incluant reprises et défigements : l'auteur américain Mark Twain est le premier emprunteur de cette chaîne et le journaliste, qui traduit en français l'aphorisation, participe à son tour à une certaine forme de défigement.

\section{Conclusion}

En reproduisant les paroles d'un locuteur rapporté, le journaliste est un acteur majeur dans la circulation des discours, qui peuvent être déformés au gré de leurs multiples réénonciations. En effet, les citations circulant dans l'espace médiatique sont propices à des opérations de défigement qui opèrent au fil du temps sous l'effet de leurs reprises successives. Par leur nature même, les petites phrases sont particulièrement appréciées par les journalistes en vue d’éventuels détournements parodiques et humoristiques.

Le locuteur emprunteur exploite généralement un Thesaurus cognitif dans lequel il sélectionne une aphorisation qu'il va dupliquer et/ou défiger. Ce Thesaurus est caractérisé par une remarquable hétérogénéité car le locuteur « défigeur » peut emprunter non seulement dans le patrimoine historique et culturel de sa propre langue mais il peut également choisir de défiger une aphorisation allogène, dans un processus sémiotique qui s’affranchit des frontières énonciatives, génériques mais aussi physiques.

En mettant en œuvre une opération de défigement, le journaliste-locuteur défigeur poursuit différentes visées. Il peut participer à la diffusion d'une aphorisation défigée à travers le temps historique, l'espace géographique et favoriser ainsi les transferts culturels et langagiers entre diverses sociétés. Les détournements opérés peuvent aussi bien affecter le contenu propositionnel aphorisé que la nature de la source énonciative censée prendre en charge l’aphorisation altérée.

Tout comme les aphorisations historiques qui circulent dans la presse, les parémies sont particulièrement propices à des défigements. En s'écartant de son contexte initial d'apparition, l'aphorisation défigée gagne en universalité et révèle en contre-point la singularité de l'écriture journalistique et le style propre au journaliste emprunteur grâce à divers degrés d’investissement du journaliste dans l'opération de défigement.

\section{Références bibliographiques}

\section{Articles de presse}

Le Monde (quotidien, cahiers et site Internet lemonde.fr)

\section{Euvres}

Molière. (1669). Le Tartuffe ou L'imposteur. Paris: Jean Ribou. Bibliothèque nationale de France. <http://gallica.bnf.fr/ark:/12148/bpt6k701569>. Consulté le 27 février 2016.

Rabelais, F. (1542). La Vie très horrificque du grand Gargantua, père de Pantagruel, jadis composée par M. Alcofribas, abstracteur de quinte essence. Livre plein de pantagruélisme. Lyon : François Juste. Bibliothèque nationale de France. <http://gallica.bnf.fr/ark:/12148/bpt6k1040342n>. Consulté le 13 août 2015.

\section{Articles et ouvrages}

Anscombre, J.-C. (2000). Parole proverbiale et structures métriques. Langages, n¹39, 6-26.

Anscombre, J.-C. et Mejri, S. (éd.). (2011). Le figement linguistique : la parole entravée. Paris : Honoré Champion. 
Brasart, P. (1994). Petites phrases et grands discours (Sur quelques problèmes de l'écoute du genre délibératif sous la Révolution française). Mots, $n^{\circ} 40$, 106-112.

Bres, J. (2014). Dialogisme, médiativité : le jeu dialogique du futur et du conditionnel français dans le marquage d'une source indirecte par ouï-dire et par conjecture. In Anscombre, J.-C., Oppermann-Marsaux, E. et Rodríguez Somolinos, A. (éd.), Médiativité, polyphonie et modalité en français, Paris : Presses Sorbonne Nouvelle, 19-34.

Bres, J. et Verine, B. (2002). Le bruissement des voix dans le discours : dialogisme et discours rapporté. In Rosier, L. (éd.), Le discours rapporté, Faits de Langues, n 19, 159-169.

Calabrese, L. (2010). Décoder les titres de presse. Les compétences de lecture et les routines rédactionnelles en question. Recherches en communication, $n$ 33, 115-129.

Courtine, J.-J. (1981). Quelques problèmes théoriques et méthodologiques en analyse du discours. À propos du discours communiste adressé aux chrétiens. Langages, $n^{\circ} 62$, 9-128.

Damourette, J. et Pichon, É. (1936). Des mots à la pensée - Essai de grammaire de la langue française 1911-1936. Tome V. Paris : d'Artrey.

Dendale, P. et Kreutz, P. (2014). Comment opposer les deux conditionnels épistémiques au conditionnel d'éventualité au moyen des notions de réalité, factualité, modalité et prise en charge? In Moreau, C., Albrespit, J. et Lambert, F. (éd.), Du réel à l'irréel 1. Diversité des langues et représentations métalinguistiques, Rennes: Presses Universitaires de Rennes, 129-151.

Fiala, P. et Habert, B. (1989). La langue de bois en éclat : les défigements dans les titres de la presse quotidienne française. Mots, $n^{\circ} 21$, 83-99.

Galisson, R. (1993). Les palimpsestes verbaux : des révélateurs culturels remarquables, mais peu remarqués... Repères, $n^{\circ} 8$, 41-63.

Krieg-Planque, A. (2009). La notion de "formule » en analyse du discours : cadre théorique et méthodologique. Besançon : Presses universitaires de Franche-Comté.

Krieg-Planque, A. (2011). Les " petites phrases » : un objet pour l'analyse des discours politiques et médiatiques. In Krieg-Planque, A. et Ollivier-Yaniv, C. (éd.), Les « petites phrases » en politique, Communication \& Langages, $n^{\circ} 168,23-41$.

Krieg-Planque, A. (2012). Analyser les discours institutionnels. Paris : Armand Colin.

Krieg-Planque, A. (2015). Construire et déconstruire l'autorité en discours. Le figement discursif et sa subversion. Mots. Les langages du politique, $n^{\circ} 107,115-131$.

Krieg-Planque, A. et Ollivier-Yaniv, C. (2011). Poser les « petites phrases » comme objet d'étude. In Krieg-Planque, A. et Ollivier-Yaniv, C. (éd.), Les « petites phrases » en politique, Communication \& Langages, n¹68, 17-22.

Lacaze, G. (2011). De l'incise au segment contextualisant : un changement d'horizon dans l'introduction du discours direct. Études de Stylistique Anglaise, $n^{\circ} 1,25-44$

Le Querler, N. (2014). Les tiroirs en -rais chez Damourette et Pichon : la notion de toncal, révélateur d'un continuum pour les valeurs du conditionnel en français? In Moreau, C., Albrespit, J. et Lambert, F. (éd.), Du réel à l'irréel 1. Diversité des langues et représentations métalinguistiques, Travaux linguistiques du CerLiCO, $n^{\circ} 25$, Rennes : Presses Universitaires de Rennes. 87-103.

Leroy, S. (2005). Le détournement dans les titres de presse : un marquage dialogique ? In Bres, J. et al. (éd.), Dialogisme et polyphonie : approches linguistiques, Bruxelles : De Boeck, 201-214.

Londei, D., Moirand, S., Reboul-Touré, S. et Reggiani, L. (éd.). (2013). Dire l'événement : langage, mémoire, société. Paris : Presses Sorbonne Nouvelle.

Maingueneau, D. (2004). Hyperénonciateur et « particitation ». Langages, n 156, 111-126.

Maingueneau, D. (2006). Les énoncés détachés dans la presse écrite. De la surassertion à l'aphorisation. TRANEL, n 44, 107-120.

Maingueneau, D. (2012). Les phrases sans texte. Paris : Armand Colin.

Maingueneau, D. (2014). Retour critique sur l'éthos. Langage et société, n 149, 31-48. 
McCallam, D. (2000). Les «petites phrases » dans la politique anglo-saxonne. Communication et langages, $n^{\circ} 126$, 52-59.

Moirand, S. (2007). Les discours de la presse quotidienne. Observer, analyser, comprendre. Paris : PUF.

Neveu, É. et Quéré L. (1996). Présentation. In Neveu, É. et Quéré, L. (éd.), Le temps de l'événement I, Réseaux, n75, 7-21.

Perrin, L. (2001). Figures et dénominations. Semen, $n^{\circ} 15$, 141-154.

Perrin, L. (éd.). (2013). Le figement en débat. Pratiques, n¹59-160.

Press Conference after Dublin European Council. Margaret Thatcher Foundation. $<$ http://www.margaretthatcher.org/document/104180>. Consulté le 30 octobre 2015.

Quéré, L. (2013). Les formes de l’événement. In Ballardini, E., Pederzoli, R., Reboul-Touré, S. et Tréguer-Felten, G. (éd.), Les facettes de l'événement : des formes aux signes, mediAzioni, $n^{\circ} 15$, <http://mediazioni.sitlec.unibo.it>. Consulté le 30 octobre 2015.

Quitout, M. (éd.). (2002). Proverbes et énoncés sentencieux. Paris : L’Harmattan.

Rastier, F. (1997). Défigements sémantiques en contexte. In Martins-Baltar, M. (éd.), La locution entre langue et usages, coll. Signes, Fontenay / Saint Cloud : ENS Editions, 307-332.

Rosier, L. (2005). Chaînes d'énonciateurs et modes d'organisation textuels : du discours rapporté à la circulation re-marquée des discours. Cahiers de praxématique, n 45, 103-124.

Rosier, L. (2008). Le discours rapporté en français. Paris : Ophrys.

Tournier. J. (1993). Précis de lexicologie anglaise. $3^{\mathrm{e}}$ édition. Paris : Nathan.

Wolton, D. (1995). La communication politique. Entre l'impensé, les a priori et les typologies. Hermès, La Revue, $n^{\circ} 17-18,9-13$.

\section{Sites Internet}

Archive Internet des marxistes. <https://www.marxists.org/francais/lenin/works/1917/10/vil19171017.htm>. Consulté le 5 octobre 2015.

В.И. Ленин Полное Собрание Сочинений том 35 В. И. ЛЕНИН стр. 418. <http://leninvi.com/t35/p418>. Consulté le 5 octobre 2015.

Wikipedia. <https://en.wikipedia.org/wiki/Lies,_damned_lies,_and_statistics>. Consulté le 28 octobre 2015.

Wikipedia. <https://en.wikipedia.org/wiki/Standing_on_the_shoulders_of_giants>. Consulté le 5 octobre 2015.

\footnotetext{
${ }^{1}$ D. Maingueneau (2004 : 112) est à l'initiative de la création de ce «mot-valise » combinant «participation » et «citation». Une particitation est «un énoncé autonome» et cette citation est «reconnue comme telle par les allocutaires, sans que le locuteur citant indique sa source » (Maingueneau, 2004 : 112).

${ }^{2}$ Comme l’évoque D. Maingueneau (2012 : 59) : «Si la grande majorité des aphorisations secondaires s’évanouissent aussitôt que détachées, certaines entrent dans quelque mémoire collective, disponibles pour un réemploi. Elles viennent ainsi rejoindre les aphorisations primaires (proverbes, slogans, devises...), vouées à être reprises à l’intérieur d'une communauté plus ou moins vaste. ».

${ }^{3}$ Selon D. Maingueneau (2012: 61), les « aphorisations sentencieuses » incluent les différentes catégories mises en évidence par M. Quitout (2002 : 12) dans son étude sur la parémiologie : "La parémiologie compte, d’une façon générale, en occident, douze parémies : le proverbe, la sentence, la locution proverbiale, le dicton, la maxime, le slogan, l'adage, le précepte, l’aphorisme, l’apophtegme, la devise et le wellérisme » (cité par Maingueneau, 2012 : 8).

${ }^{4}$ La «mémoire discursive » est un «concept forgé par l'analyste du discours J.-J. Courtine (1981) pour rendre compte du fait qu'un énoncé peut faire appel à des énoncés antérieurs, et ce à l’insu de l'énonciateur » (Calabrese, $2010: 121)$.
} 


\footnotetext{
${ }^{5}$ L'expression mémorabilité est empruntée à D. Maingueneau (2014 : 166) qui la définit ainsi : «La "mémorabilité", le fait qu'à un moment et en un lieu donnés un énoncé puisse devenir trace, n’est pas un phénomène extérieur à l'énonciation ; celle-ci est prise dans une relation constitutive à son propre devenir-trace ».

${ }^{6}$ La petite phrase est un «énoncé détaché prêt pour la reprise » qui « est doté d’une valeur illocutoire par son détachement même, et [qui] se donne à voir comme disponible pour une construction en événement " (Krieg-Planque, $2011: 36$ ).

${ }^{7}$ Le terme circulème est emprunté à L. Rosier (2005 : 104).

${ }^{8}$ L'expression pulsion ludique est empruntée à J. Tournier (1993 : 168) qui envisage les divers ressorts de la création lexicale.

${ }^{9}$ D. Wolton (1995 : 9) emploie cette expression pour caractériser l'activité politique.

${ }^{10}$ L’expression énoncé aphorisé est empruntée à D. Maingueneau (2006 : 117).

${ }^{11}$ La citation reformulée l'a été en fonction de contraintes de «concision », comme c'est aussi le cas d'une « formule » (voir Krieg-Planque, 2009 : 73-75).

${ }^{12}$ Voir l'article de D. McCallam (2000) consacré aux petites phrases dans la politique anglo-saxonne.

${ }^{13}$ Source : Wikipedia (<https://en.wikipedia.org/wiki/Standing_on_the_shoulders_of_giants>).

14 Source : $\quad$ site Inchive desnet marxistes
<https://www.marxists.org/francais/lenin/works/1917/10/vil19171017.htm>.

15 Source : site В.И. Ленин Полное Собрание Сочинений том 35 В. И. ЛЕНИН стр. 418. $<$ http://leninvi.com/t35/p418>.

${ }^{16}$ Ce verbe introducteur atypique précise le medium de communication utilisé par l'aviateur pour transmettre son message.

${ }^{17}$ Pour la définition d’un « segment contextualisant », voir l’étude de G. Lacaze (2011).

${ }^{18}$ Voir l'étude de N. Le Querler (2014) sur l’étude des tiroirs en -rais selon Damourette et Pichon.

${ }^{19}$ Source : Wikipedia (<https://en.wikipedia.org/wiki/Lies,_damned_lies,_and_statistics>).
} 